\title{
Acoustical Coupling of Lizard Eardrums
}

\author{
Jakob Christensen-Dalsgaard ${ }^{1}$ and Geoffrey A. Manley ${ }^{2}$ \\ ${ }^{1}$ Institute of Biology, University of Southern Denmark, Campusvej 55, 5230, Odense M, Denmark \\ ${ }^{2}$ Lehrstuhl für Zoologie, Technische Universität München, Hochfeldweg 2, 85350, Freising, Germany
}

Received: 10 September 2007; Accepted: 25 June 2008; Online publication: 22 July 2008

\begin{abstract}
Lizard ears are clear examples of two-input pressuredifference receivers, with up to 40-dB differences in eardrum vibration amplitude in response to ipsi- and contralateral stimulus directions. The directionality is created by acoustical coupling of the eardrums and interaction of the direct and indirect sound components on the eardrum. The ensuing pressure-difference characteristics generate the highest directionality of any similar-sized terrestrial vertebrate ear. The aim of the present study was to measure the gain of the direct and indirect sound components in three lizard species: Anolis sagrei and Basiliscus vittatus (iguanids) and Hemidactylus frenatus (gekkonid) by laser vibrometry, using either free-field sound or a headphone and coupler for stimulation. The directivity of the ear of these lizards is pronounced in the frequency range from 2 to $5 \mathrm{kHz}$. The directivity is ovoidal, asymmetrical across the midline, but largely symmetrical across the interaural axis (i.e., front-back). Occlusion of the contralateral ear abolishes the directionality. We stimulated the two eardrums with a coupler close to the eardrum to measure the gain of the sound pathways. Within the frequency range of maximal directionality, the interaural transmission gain (compared to sound arriving directly) is close to or even exceeds unity, indicating a pronounced acoustical transparency of the lizard head and resonances in the interaural cavities. Our results show that the directionality of the lizard ear is caused by the acoustic interaction of the two eardrums. The results can be largely explained by a simple acoustical model based on an electrical analog circuit.
\end{abstract}

Correspondence to: Jakob Christensen-Dalsgaard · Institute of Biology University of Southern Denmark - Campusvej 55, 5230, Odense M, Denmark. Telephone: +45-65502448; fax: +45-65930457; email: JCD@biology.sdu.dk
Keywords: lizard, tympanum, vibrometry, directional, hearing, reptile

Abbreviations: IL - ipsilateral; CL - contralateral

\section{INTRODUCTION}

The ability to locate sound sources is a fundamentally important task of any hearing animal. Mammals have ears that act as independent pressure receivers and therefore are inherently non-directional. The same is true of some birds such as the barn owl in the higherfrequency ranges. The strategy of these organisms has been to exploit the sound diffraction cues generated by peripheral structures (outer ears) and the interaural time differences generated by sound propagation around the head. In mammals and birds, processing of parameters associated with interaural time and intensity differences is a major feature of the auditory pathway in the most extreme cases (e.g., barn owl), leading to specializations at every level of the auditory processing chain (Christensen-Dalsgaard 2005).

However, other terrestrial vertebrates have ears that are inherently directional even at low frequencies and in the absence of peripheral diffracting structures. These animals - lizards, frogs, and birds-have ears that are functionally acoustically connected through the mouth cavity and/or interaural canal. The acoustical connection allows sound to reach both sides of the eardrum. The eardrum motion is thus driven by the instantaneous pressure difference between the external and internal sound components and reflects the phase and amplitude differences between these components caused by sound propagation, filtering by outer and middle ears, and transmission through internal pathways. In these animals, part of the binaural interaction takes place directly on the eardrum. This results in pressure-difference receiver 
operation of the ear and high directionality of the eardrum, provided the interaural coupling is sufficiently strong. It is important to note that the existence of an interaural connection does not in itself provide for a useful directionality. If sound transmitted from the contralateral ear is strongly attenuated (i.e., the interaural transmission gain is low), the resulting directionality will be small. If the external and internal sound components have equal amplitudes, the eardrum response can range from total cancellation to a doubling of the effective sound pressure difference driving the eardrum, depending on the phase difference between direct and indirect components (Klump 2000; Feng and Christensen-Dalsgaard 2007). If the contralateral sound is attenuated by $6 \mathrm{~dB}$ during interaural transmission, the effect of interaural interaction ranges from -6 to $4 \mathrm{~dB}$, giving the maximal directional difference of $10 \mathrm{~dB}$ found in frogs (Christensen-Dalsgaard 2005; Ho and Narins 2006) and birds (Larsen et al. 2006). Furthermore, the interaural transmission gain will be frequency dependent, and even in cases where the interaural canal is large and attenuation low in a certain frequency range, this frequency range may be outside the range of interest for the animal. This is the case for the barn owl that has a large interaural canal that provides directionality at low frequencies but attenuates the high frequencies used by the barn owl for sound localization. In the behaviorally important frequency range, the barn owl ears are therefore functionally independent pressure receivers (Moiseff and Konishi 1981).

Recent biophysical measurements show that the lizard ear is highly directional. Measurements of eardrum vibrations in response to free-field sound have shown that the ear exhibits a robust directionality in a 2-kHz-wide band, with up to 40-dB differences between responses to ipsi- and contralateral stimulation. Furthermore, the directional characteristics of the eardrum are asymmetrical across the midline and will therefore be sharpened by neural binaural comparisons in the central nervous system (CNS; Christensen-Dalsgaard and Manley 2005). The directionality can be abolished by occluding one eardrum and therefore depends on acoustical coupling of the eardrums, i.e., on acoustical interference between the direct and indirect sound component at the eardrum.

These experiments strongly suggested that the main inputs to lizard directional hearing are the two eardrums. In the present study, we have quantified the acoustical interference of the eardrums across the head and also investigated another putative acoustical input, the nares. Finally, the response of the ear is compared to two models of the two-input ear, a simple calculation based on summation of the direct and the indirect input at the eardrum, and a real acoustical model based on the electrical analog circuit of the ear.

\section{METHODS}

We investigated the directionality of the ear of three lizard species: three Anolis sagrei and two Basiliscus vittatus (both iguanids) and three Hemidactylus frenatus (gekkonid) by laser vibrometry measurements of eardrum vibrations. The animals were lightly anesthetized by inhalation of isoflurane $\left(1-2 \%\right.$ in $\left.95 \% \mathrm{O}_{2}\right)$ and were immobile with normal respiration during the experiments.

Stimulation and data recording were controlled by Tucker-Davis system 2 hardware and customized software (DragonQuest). Stimuli were frequency sweeps (175 ms, 200-7,500 Hz, 16 sweeps, levels of 80 to $90 \mathrm{~dB}$ SPL) emitted in an anechoic room from 12 JBL $1 G$ loudspeakers placed at $30^{\circ}$ intervals around the lizard, each at 1-m distance. The room has been tested to be anechoic to below $200 \mathrm{~Hz}$. However, some reflections, especially from the laser setup, are probably unavoidable and may explain the spectral ripple in some of the measurements. The signal sent to the loudspeakers was deconvoluted for the individual loudspeaker (measured with a B\&K 0.5" microphone at the center of the setup before placing the animal) by dividing the spectrum of the sweep by the transfer function of the speaker. For local ipsi- and contralateral stimulation, we used a Beyer DT-48A headphone in a coupler placed approximately $0.5 \mathrm{~cm}$ from the eardrum but not sealing the eardrum. With both free-field and local stimulation, the sound at the animal's eardrum was measured with a B\&K 4182 probe microphone, digitized (22 kHz sample rate, 8,192 samples) using the TDT AD-converter (AD2), and stored in a PC. For the local stimulation, the nonstimulated ear was partially shielded using Vaseline barriers, and the direct sound transmission around the head was measured with the probe microphone at the non-stimulated ear and found to be reduced by at least $20 \mathrm{~dB}$. Eardrum vibrations were measured by a Dantec laser doppler vibrometer, and we obtained strong reflections directly (no added reflector) from the tip of the extracolumellar attachment close to the center of the eardrum. Sound and laser recordings were averaged over 16 presentations.

\section{DATA ANALYSIS}

The quality of the measurements was gauged by calculating the coherence function (the fraction of output power attributed to the stimulus signal), and only measurement values where the coherence function was close to 1 are reported here.

\section{Cylinder surface plots}

The eardrum vibrations are displayed as cylinder surface plots as described by Christensen-Dalsgaard 
and Manley (2005). Cylinder surface plots are interpolated contour plots of amplitude with direction $(X$, 12 directions) and frequency ( $Y, 500$ frequency bands) as independent variables. Each horizontal line corresponds to a polar plot, and each vertical line corresponds to an amplitude spectrum of eardrum motion stimulated by sound from a certain direction. Following Christensen-Dalsgaard and Manley (2005), we calculated the directional bandwidth from the spectra as the frequency band where the response to ipsi- and contralateral free-field stimulation differed by more than $3 \mathrm{~dB}$. We used the interaural vibration amplitude difference function (Jørgensen et al. 1991) as a simple model of binaural processing to predict the output of a simple model neuron that is excited by the ipsilateral ear and inhibited by the contralateral ear (an EI neuron; Goldberg and Brown 1969). The function computes the vibration amplitude difference (in decibel) between the input from the ipsi- and contralateral ear by subtracting the free-field eardrum vibration transfer function (in decibel) by its reflection along the frontal caudal axis and assumes that the directionalities of the two ears are identical. Subtraction of decibel values really corresponds to division but is assumed to reflect the neural processing since within a neuron's dynamic range, spike rate is a linear function of the level in decibel.

\section{Interaural transmission gain and free-field reconstruction}

Using a method described by Michelsen and Rohrseitz (1995), the sound arriving at the two surfaces of the eardrum can be calculated from local stimulation data. Briefly, if the eardrum vibration response to local sound source stimulation is measured, the IL eardrum transfer functions to IL and CL stimulation can be used to measure the sound arriving at the internal surface of the eardrum: The ratio between the CL and IL transfer functions is the gain of the interaural transmission pathway, under the assumption that the direct sound transmission from the stimulated to the non-stimulated ear is insignificant (see Fig. 4).

If the free-field response is generated by the two tympanic inputs, it should then be possible to reconstruct the free-field response by calculating the difference between direct and indirect sound at the eardrum and multiply it by the eardrum transfer function. The procedure (based on Michelsen and Rohrseitz 1995) uses the following steps:

1. Measure the transfer functions of one eardrum to IL and CL stimulation as above and calculate the interaural transmission gain.

2. Measure the sound field at the two eardrums.
3. Multiply the sound field at the contralateral eardrum by the interaural transmission gain to calculate the sound arriving at the internal surface of the eardrum.

4. Subtract the two sound components (vectorially).

5. Multiply the pressure difference by the eardrum transfer function. This is the free-field response of the eardrum, assuming that the two eardrums are the only sound inputs (and allowing for measurement noise).

\section{Model}

Assuming that the lizard ear is a two-input system, it can be modeled by a very simple lumped-element electrical analog (Fletcher 1992; Christensen-Dalsgaard and Manley 2005) consisting of the two sound inputs and three impedances: one for each tympanum $\left(Z_{T}\right)$ and one for the buccal and mouth cavity $\left(Z_{V}\right)$. Diffraction effects are neglected, so the two sound inputs $\left(p_{1}\right.$ and $\left.p_{2}\right)$ differ only in phase by

$$
\Delta \varphi=\frac{\omega}{c} \cdot d \cdot \sin \theta
$$

where $\omega$ is the angular frequency, $c$ the velocity of sound, $d$ the interaural distance, and $\theta$ the sound incidence angle relative to the body axis. The circuit equation is $U_{1}=\frac{p_{1}\left(Z_{\mathrm{T}}+Z_{\mathrm{V}}\right)-p_{2} Z_{\mathrm{V}}}{Z_{\mathrm{T}}\left(Z_{\mathrm{T}}+2 Z_{\mathrm{V}}\right)}$ (Fletcher 1992), where $U_{1}$ is the volume velocity of one of the tympana, so $U_{1}$ divided by the tympanum area is the model vibration velocity of the tympanum.

The buccal volume $\mathrm{V}$ was measured from casts of the mouth cavity, and the cavity impedance was calculated from the volume as $Z_{V}=\frac{1}{i \omega \frac{V}{\rho c^{2}}}$. To calculate the tympanic impedance $Z_{\mathrm{T}}$, we observe that the local stimulation experiment, where the contralateral ear is stimulated and the vibrations are measured at the ipsilateral ear, corresponds to $p_{1}=0$ in the circuit, and therefore $Z_{\mathrm{T}} U_{1}+Z_{\mathrm{V}}\left(U_{1}+U_{2}\right)=p_{1}=0$ or $Z_{\mathrm{T}}=-Z_{\mathrm{V}}\left(1+\frac{U_{2}}{U_{1}}\right)$

$\frac{U_{2}}{U_{1}}$, the ratio of the vibration of the two eardrums, is the interaural gain $G$ measured in the local stimulation experiments, assuming that the two eardrums have identical transfer functions. Therefore, $Z_{T}$ can be calculated as $Z_{\mathrm{T}}=-Z_{\mathrm{V}}(1+G)$, where $G$ is the interaural transmission gain (after Fletcher 1992).

\section{RESULTS}

The three species varied in size and head dimensions. Anolis and Hemidactylus measured 50-60 mm snout-vent, weight 1-3 g, whereas Basiliscus measured $130 \mathrm{~mm}$ and weighed $79-81 \mathrm{~g}$. The head width was approximately $10 \mathrm{~mm}$ in Hemidactylus, $7 \mathrm{~mm}$ in Anolis, and $20 \mathrm{~mm}$ in 
Basiliscus. Finally, the mouth and buccal cavity volume was estimated from casts: $0.064 \mathrm{~cm}^{3}$ in Anolis, $0.32 \mathrm{~cm}^{3}$ in Hemidactylus, and $2.5 \mathrm{~cm}^{3}$ in Basiliscus.

\section{Eardrum directionality}

The eardrum of all the lizards exhibited strong directionality as shown by the cylinder surface plots in Figure 1A (upper row). In all three species, the directional characteristics are strongly asymmetrical along the frontal angles but largely symmetrical across the interaural axis (i.e., front-back). The gradient along the frontal angles is steep, up to $40 \mathrm{~dB}$ from $-60^{\circ}$ to $60^{\circ}$. The directional bandwidth (as defined in "Methods") was very different in the three species: $0.7 \mathrm{kHz}$ in Basiliscus, $4.9 \mathrm{kHz}$ in Hemidactylus, and $>6 \mathrm{kHz}$ in Anolis, and the peak frequency of the eardrum was approximately at $2-4 \mathrm{kHz}$ for all three species. The maximal directionality was $40 \mathrm{~dB}$ in Anolis, $36 \mathrm{~dB}$ in Hemidactylus, and $30 \mathrm{~dB}$ in Basiliscus. The directionality was abolished when the contralateral eardrum was enclosed by a dome of Vaseline (see below, Fig. 3B). In all three species, diffraction effects were relatively small at the frequencies studied (data not shown) but increased systematically with frequency. In the larger Basiliscus, the low-frequency limit of diffraction, defined as a 3-dB difference between IL and CL sound incidence, was found at $2.7 \mathrm{kHz}$. In the smaller lizards, this diffraction limit was higher, $4.2 \mathrm{kHz}$ in Anolis and $4.3 \mathrm{kHz}$ in Hemidactylus.

Assuming that the lizard CNS computes the difference between the binaural inputs (for example in EI neurons in the brain stem), any directivity pattern that is asymmetrical around the midline is sharpened. In Figure 1B (lower row), the neural subtraction is 'modeled' by subtracting the eardrum

A

\section{frequency $(\mathrm{Hz})$}

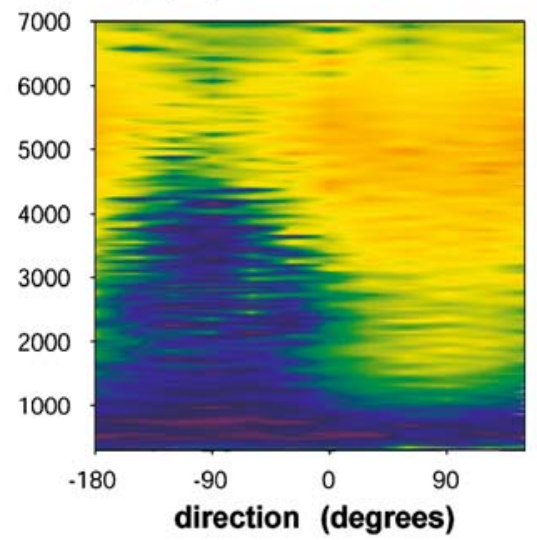

B

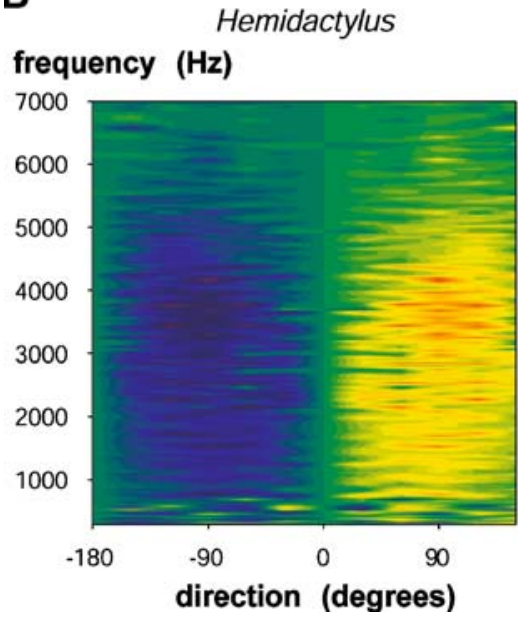

Anolis

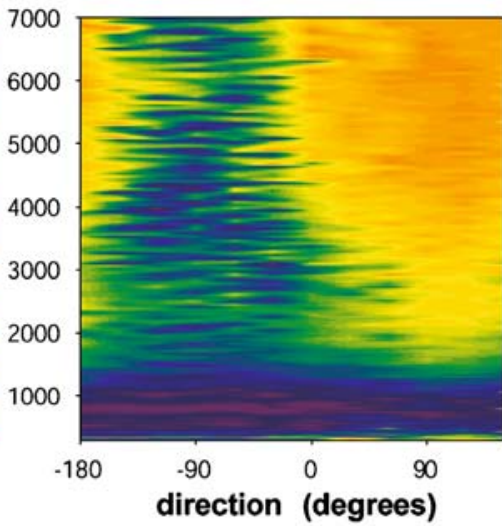

Anolis

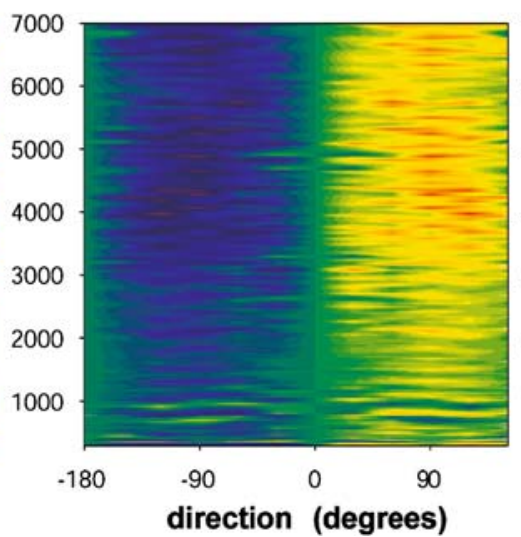

Basiliscus

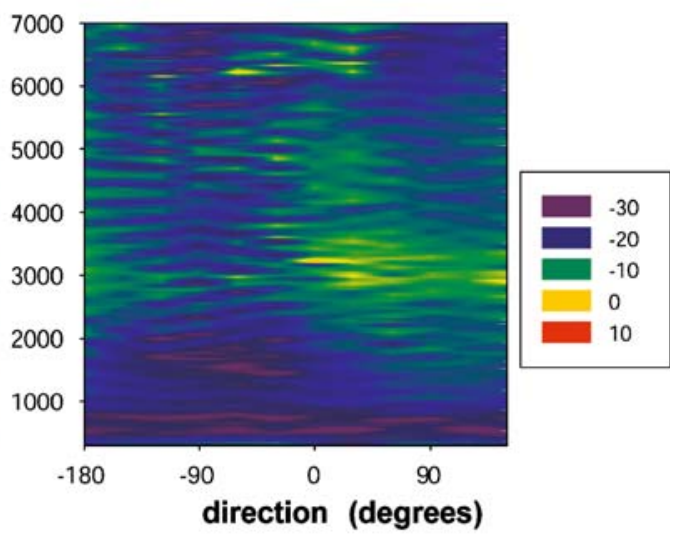

Basiliscus

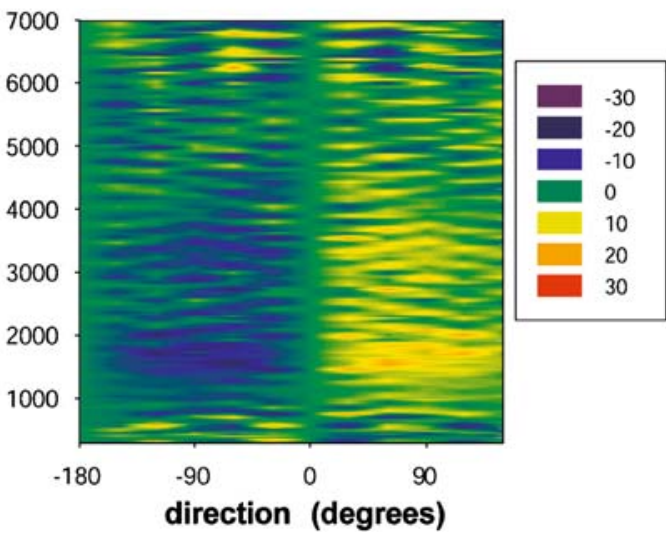

FIG. 1. A Eardrum vibration amplitude as a function of sound direction in three lizard species: the gecko Hemidactylus (left), anole Anolis (middle), and basilisk Basiliscus (right). $X$ axis Direction angle in degrees (ipsilateral directions are positive, contralateral negative, $0^{\circ}$ is frontal); $Y$ axis frequency (hertz). The color scale is the eardrum vibration amplitude transfer function (decibel re. $1 \mathrm{~mm} / \mathrm{s} / \mathrm{Pa}$ ). B The lower row shows interaural vibration difference (see 'Methods' for details) in Anolis (left), Hemidactylus (middle), and Basiliscus (right). $X$ axis direction; $Y$ axis frequency; color scale is interaural difference (decibel). 
directivity (i.e., free-field eardrum transfer function, in decibel) by its mirror reflection along the midline (Jørgensen et al. 1991). In the three lizard species, this computation leads to a steep gradient, especially in the frontal directions. In Hemidactylus and Anolis, the computation generates a large directional difference through most of the hearing range and very robust lateralization. In Basiliscus, however, the directional sharpening is confined to a frequency band extending from 1 to $4 \mathrm{kHz}$, reflecting the narrower directional bandwidth in this species.

The directivity shows some individual variation within the species as well (Fig. 2). Generally, the response to IL stimulation and the maximal directional difference is similar within individuals, but the peak directional frequency shows individual variation. The two smaller species (Hemidactylus and Anolis) have similar maximal sensitivities (approximately $1 \mathrm{~mm} / \mathrm{s} / \mathrm{Pa}$ ), whereas Basiliscus is less sensitive $(0.3 \mathrm{~mm} / \mathrm{s} / \mathrm{Pa})$.

\section{Blocking the nares}

The nares are putative sound inputs, and we investigated the effect of blocking only one of them (in order not to interfere with breathing). To highlight the possible effects, we also blocked the contralateral eardrum. Figure 3 shows the normal directivity plot (a) compared to CL eardrum block (b) and the ratio between CL block and CL + nares block (c). The directivity is largely abolished by blocking the contralateral eardrum, and the additional effect of blocking the nares is generally below $2 \mathrm{~dB}$ and mainly seen at relatively low frequencies $(1-2 \mathrm{kHz})$, where the freefield directionality and sensitivity of the eardrum is small.

\section{Interaural transmission gain}

Transmission gain for the interaural sound component can be measured as the ratio of the eardrum vibrations in response to contra- and ipsilateral local stimulation (Michelsen and Rohrseitz 1995, see "Methods" for details), under the assumption that direct sound transmission from the stimulated to the non-stimulated ear is insignificant. In all cases, this sound component was $20-30 \mathrm{~dB}$ or more below the level at the stimulation site. The significance of this component was investigated by measuring the sound transmitted to the non-stimulated ear and correcting the transfer function by subtracting the transmitted sound component vectorially from the sound spectrum at the stimulation site. An example of this correction is shown for Hemidactylus in Figure 4. The corrected transfer function is at most decreased by $1-$ $2 \mathrm{~dB}$ compared to the uncorrected transfer function.

In Anolis and Hemidactylus, interaural transmission gains are close to or higher than $0 \mathrm{~dB}$ at frequencies around $2-5 \mathrm{kHz}$, the region of maximal directionality (Fig. 5). At frequencies below $1.5 \mathrm{kHz}$, the transmission gain is dominated by measurement noise. The phase of the transmission gain (Fig. 5B) generally shows a linear phase-frequency relationship with a slope of -0.4 to $-1.1 \mathrm{rad} / \mathrm{kHz}$, corresponding to an interaural delay of 70 to $185 \mu \mathrm{s}$.

In contrast, as shown in Figure 5, the transmission gain in Basiliscus is much lower. The peak is at $0.7 \mathrm{kHz}$ $(-5 \mathrm{~dB})$, the gain is approx. $-20 \mathrm{~dB}$ at frequencies above $2 \mathrm{kHz}$, and the signal/noise ratio of the measurement is therefore less favorable (note that the gain curve is smoothed). However, a part of the phase-frequency curve in Basiliscus $(1.5$ to $3 \mathrm{kHz}$, the range of maximal directionality) can be fitted with a line having a slope of $-1.2 \mathrm{rad} / \mathrm{kHz}$, corresponding to an interaural delay of $191 \mu \mathrm{s}$.

\section{RECONSTRUCTION}

If the eardrum free-field response is caused by interaction of the ipsi- and contralateral input, it
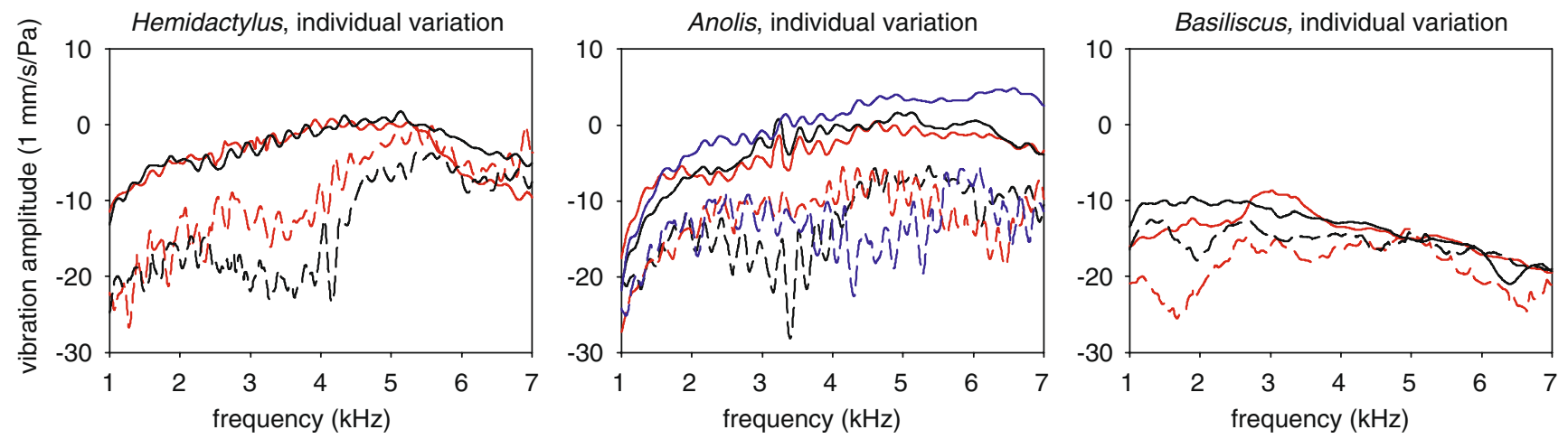

FIG. 2. Individual variation in the three species. Each figure shows the eardrum vibration transfer functions in response to free-field stimulation from IL $\left(90^{\circ}\right)$ and $\mathrm{CL}\left(-90^{\circ}\right.$, dotted lines) directions in Anolis (left, two individuals), Hemidactylus (middle, three individuals), and Basiliscus (right, two individuals). Each animal is identified by color. The CL stimulation responses are in all cases lower than IL responses. 


\section{A normal eardrum transfer function}

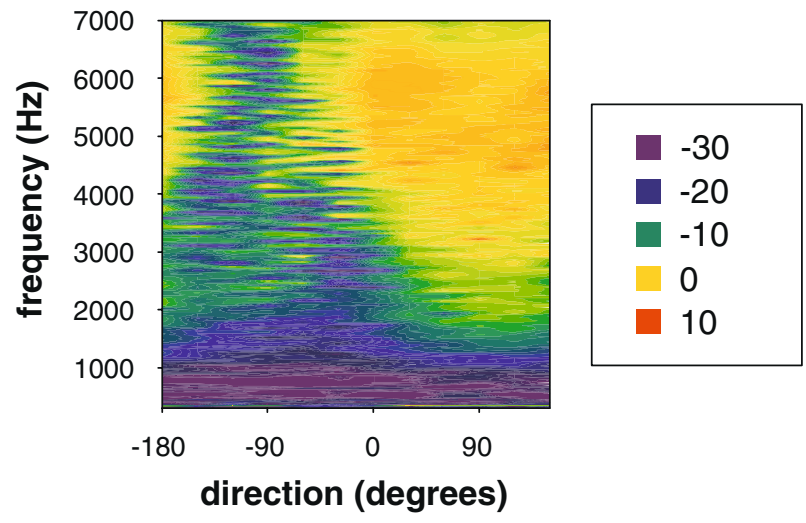

B

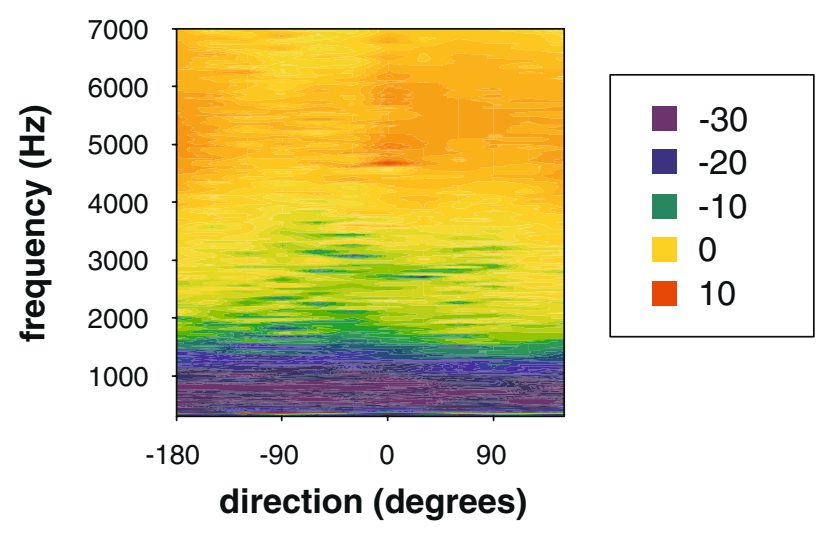

\section{CL eardrum+nares blocked}

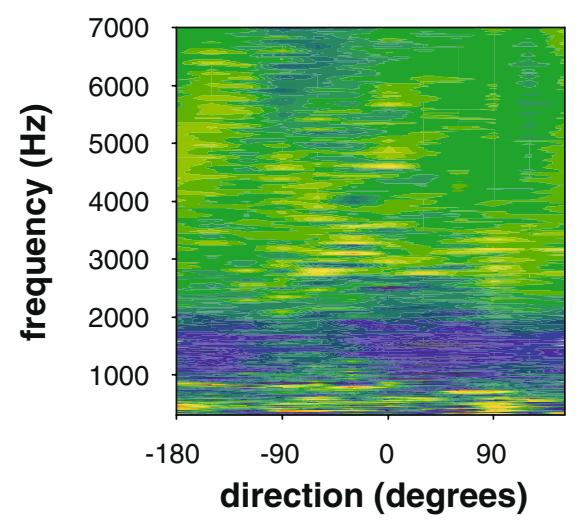

FIG. 3. Directionality of the eardrum in Hemidactylus before (A) and after (B) occluding the contralateral eardrum with a dome of Vaseline. In C, the left naris was additionally blocked with Vaseline, and the plot shows the amplitude ratio (in decibel) of nares block to the eardrum-blocked condition.

should be possible to reconstruct the free-field response of the eardrum from measurements of the sound input at the eardrum, the eardrum transfer function, and the interaural gain, as described in the "Methods".

The figures (Fig. 6B, middle row) show the result of reconstructing the free-field response of the eardrum of Hemidactylus (right) and Anolis (left). In comparison to the measured free-field response (a, top row), the reconstructions show the same region of maximal CL and IL differences, demonstrating that the ear can be reasonably well described by two sound inputs. However, especially in Anolis, the reconstruction deviates from the measured spectra. The deviations between the measured and the reconstructed spectra can be due partly to measurement noise, particularly in the very noisy phase spectrum, but it is also possible that there are additional sound inputs of relatively minor importance (nares, lungs) that contribute to the free-field response of the eardrum.

\section{Model}

We used the lumped-parameter model described previously (Fletcher 1992; Christensen-Dalsgaard and Manley 2005) but used parameters calculated from the transmission gain measurements (see "Methods" for details). The real part of the calculated tympanum impedance ranged from $10^{8}$ to $2 \times 10^{8} \mathrm{~kg} \mathrm{~m}^{-4} \mathrm{~s}^{-1}$. The model calculations (Fig. 6C, bottom row) show systematic, directional effects. Ipsilateral angles produce up to $20 \mathrm{~dB}$ higher vibration amplitudes than contralateral angles, and in Hemidactylus, the model calculations show the same frequency regions of maximal directionality, i.e., in the ranges $2-3$ and $3.5-4 \mathrm{kHz}$, as the eardrum measurements. In Anolis, the model shows maximal directionality around 4.5 to $6 \mathrm{kHz}$ but smaller directionality than that observed below $4.5 \mathrm{kHz}$. The model directionality in both

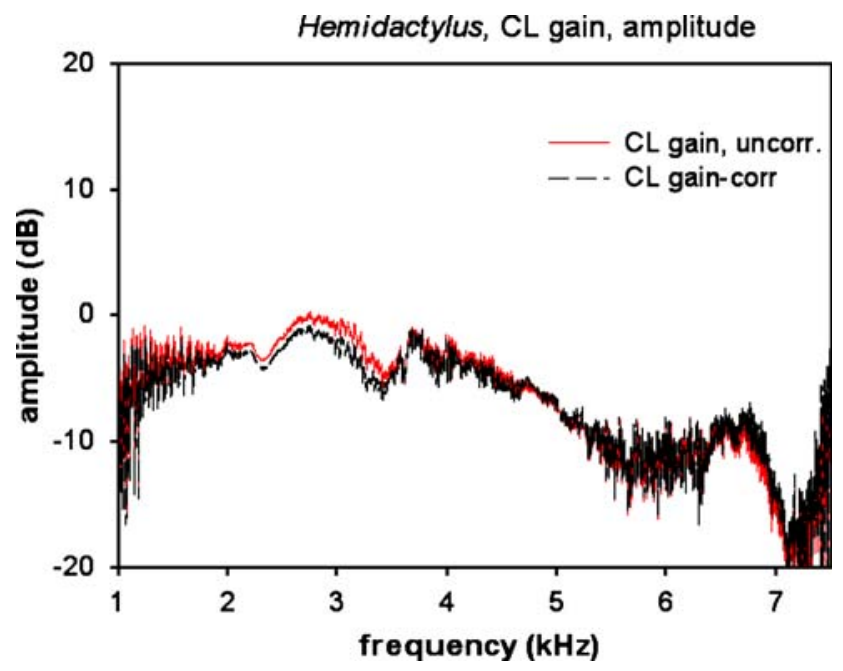

FIG. 4. Interaural transmission gain in Hemidactylus. The interaural transmission gain is calculated as the ratio of contra- and ipsilateral transfer functions, as described in "Methods". Only the amplitude spectrum is shown here. The two curves are the gain functions with (red curve) and without (black curve) correction for direct transmission of sound (see "Methods" for details). 

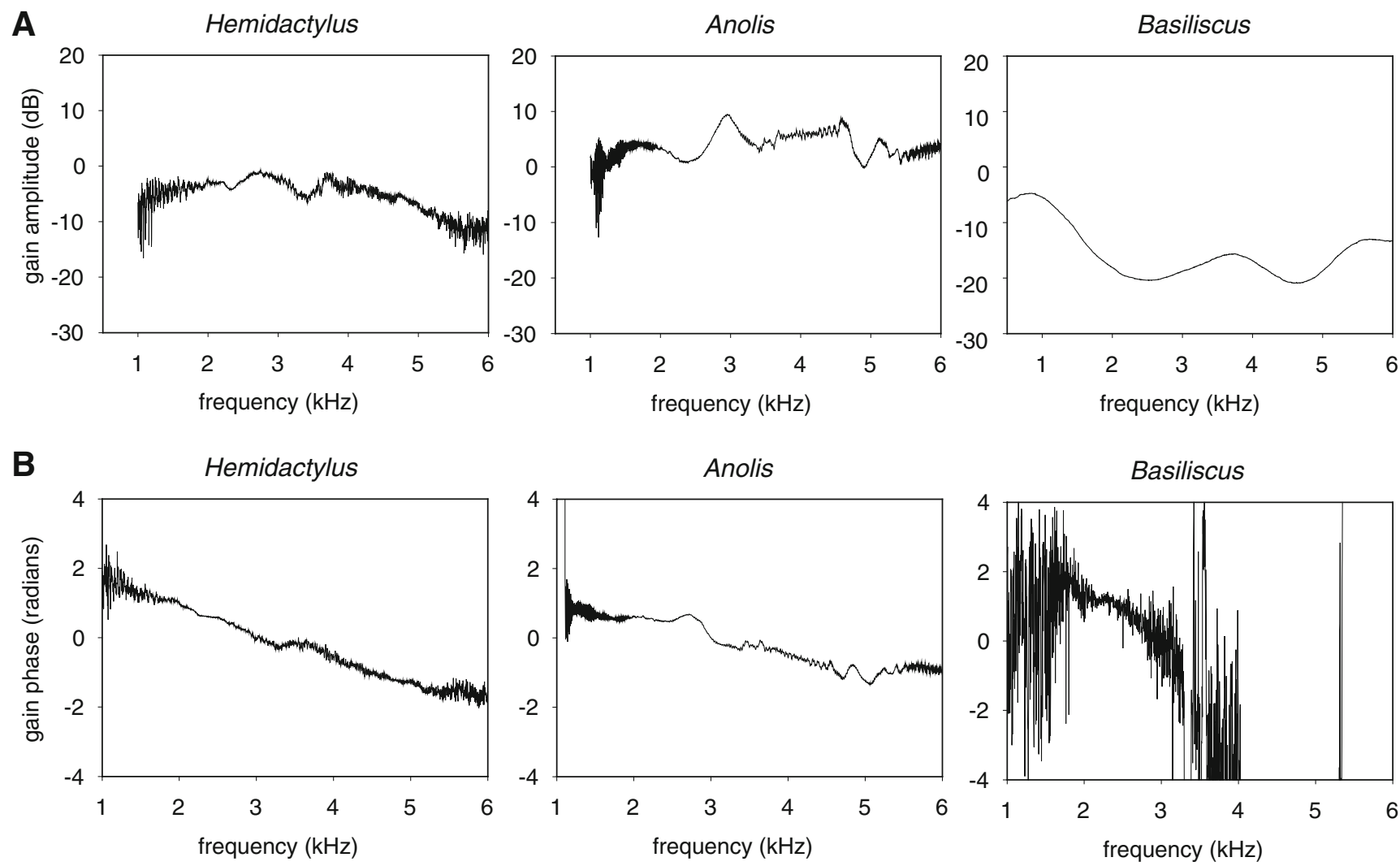

FIG. 5. Interaural transmission gains in Hemidactylus (left), Anolis (middle), and Basiliscus (right, smoothed data) calculated as the ratio of contra- and ipsilateral transfer functions. Data from individual animals, see "Methods" for details. A Amplitudes, B phases in the three lizard species.

animals shows the same regions of maximal directionality as the reconstruction (Fig. 6B).

\section{DISCUSSION}

We have shown that the lizard head is highly transparent to sound. Two of the investigated species, Anolis and Hemidactylus, showed especially strong eardrum directivity generated by interaural acoustical coupling through the mouth cavity (Fig. 1).

These species also had a large gain for interaural sound transmission (Fig. 5A). At the frequencies of highest directionality, the interaural gain exceeded $0 \mathrm{~dB}$, so sound arrives at the internal surface of the eardrum with higher amplitude than does the direct sound component at the external surface of the eardrum. The phase of the interaural gain shows a large interaural delay (Fig. 5B). From the linear phase-frequency plot, the delay estimate for the smaller lizards Anolis and Hemidactylus $(1 \mathrm{~cm}$ head width) is approximately $100 \mu$ s or three times larger than the arrival-time difference (33 $\mu$ s for a lizard with $1 \mathrm{~cm}$ head width). Furthermore, the ear conforms reasonably well to a simple, two-input model, and the main elements of the directional response can be reconstructed from two sound inputs. By comparison, in the third, larger species Basiliscus (head width $2.5 \mathrm{~cm}$ ), both directionality and interaural transmission were considerably reduced. However, also in this species, the interaural delay $(190 \mu \mathrm{s})$ is much larger than expected from arrival-time differences $(82 \mu \mathrm{s}$ at $2.5 \mathrm{~cm}$ head width, assuming no diffraction).

The directivity is comparable to the species investigated previously (Christensen-Dalsgaard and Manley 2005), but the directionality extends to higher frequencies in Anolis and Hemidactylus, probably because they are small animals and their directional bandwidth is also larger than we reported for other lizard species.

The large interaural gains in Anolis and Hemidactylus are surprising since sound must be attenuated by transmission through the CL eardrum. However, the attenuation by the eardrum is probably small since the eardrum is delicate in both species and therefore well matched to the impedance of air. Additionally, internal sound may be amplified by resonances in the interaural pathway, both the large mouth cavity and the open middle ear cavities. Resonances probably also partly explain why the gain phase shows approximately three times the delay expected from arrival-time differences in Anolis and Hemidactylus. 

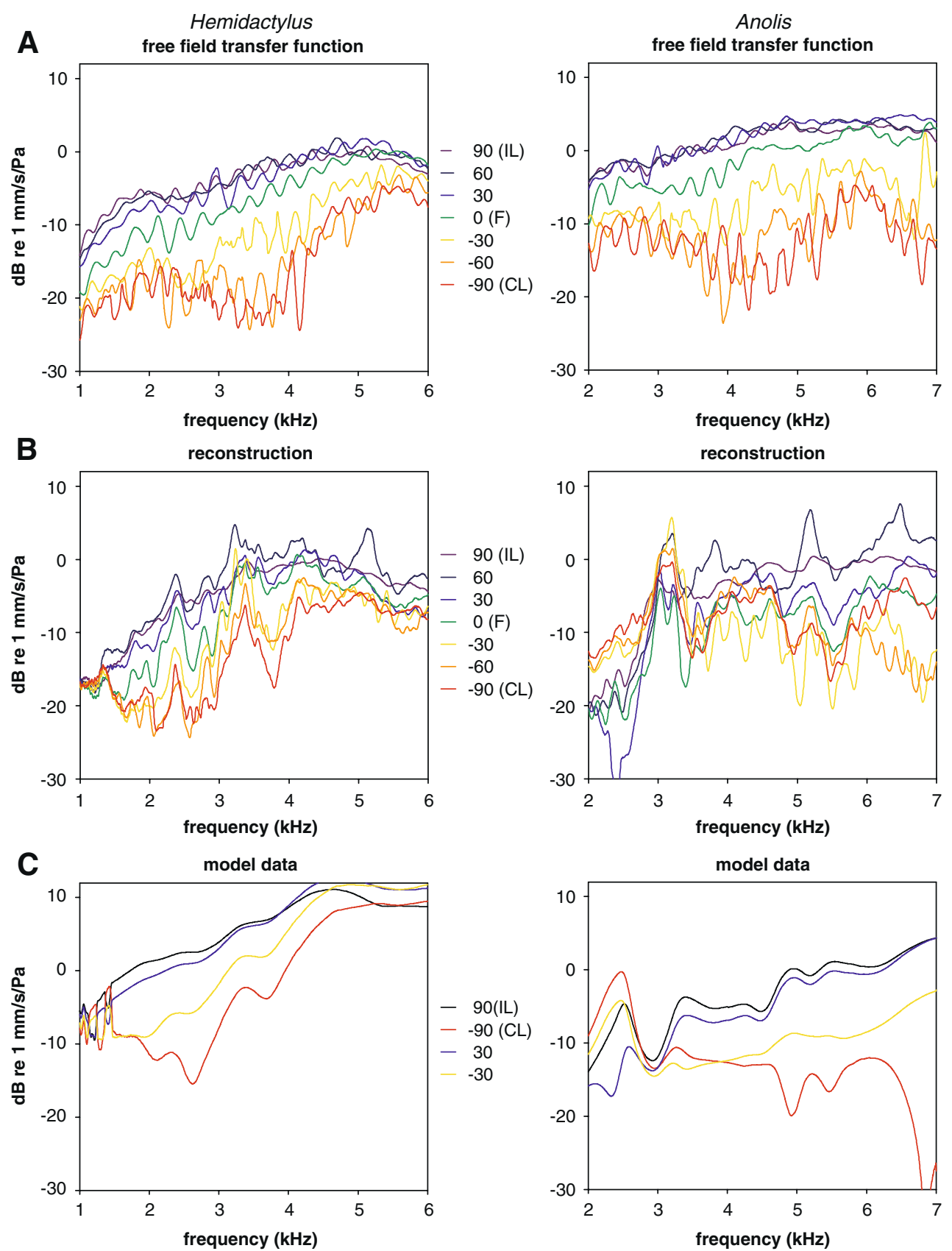

FIG. 6. Reconstruction and modeling of the free-field response of the eardrum of Hemidactylus (left) and Anolis (right). The spectra are eardrum transfer functions to sound from the seven frontal directions. For comparison, measured free-field response spectra from two individual animals are shown in the top row (A). The middle row (B) shows reconstructions, calculated as described in "Methods", from measurements in the same individuals as in $\mathbf{A}$. The bottom row $(\mathbf{C})$

Comparison of the interaural transmission gains and the directivity plots (Fig. 1) shows that the differences in directivity among the three species can be explained by differences in interaural transmission. The relatively wide-band directivity in Anolis corresponds to an interaural gain that exceeds $0 \mathrm{~dB}$ in a $3-\mathrm{kHz}$ frequency band (Fig. 5). In this species, the highest frequencies of spontaneous otoacoustic emis- shows model calculations of eardrum vibration. The eardrum impedance was calculated from the gain measurements and used in a lumpedelement model of the lizard ear (see "Methods" for details). Model data are only shown for one Hemidactylus (left) and an Anolis specimen. For Basiliscus, the transmission gain phase data (Fig. 5) are too noisy to be usable. For each animal, calculations from four directions are shown.

sions reported in any lizard have been measured (7.7 kHz, Manley and Gallo 1997), suggesting that its upper frequency-response limit is higher than in most lizard species (Manley 1990). In Hemidactylus, directionality is restricted to a $2-3-\mathrm{kHz}$ band, corresponding to an interaural gain that is more peaked and generally smaller than in Anolis. Finally, in Basiliscus, directivity is generally lower with a peak at 
2-3 kHz. The interaural gain function in this species has lower amplitude than in the other species, with a maximum at $-6 \mathrm{~dB}$ at $700 \mathrm{~Hz}$. One explanation for the lower interaural gain may be that the (more superficial) eardrum is less sensitive in this species. This explanation is supported by the 10- $\mathrm{dB}$ lower overall sensitivity in Basiliscus than in Anolis and Hemidactylus.

The directionality shows some individual variation that is probably caused by slightly different acoustical properties of the mouth cavity in different individuals since the IL responses show less individual variation than the CL responses (Fig. 2).

Recent measurements of interaural transmission in the frog Rana pipiens (Ho and Narins 2006) and the bird Melopsittacus undulatus (Larsen et al. 2006) demonstrated a maximal interaural gain of $-10 \mathrm{~dB}$. The reduced gain and resulting poorer directionality in the frog and bird studied are probably caused by attenuation (by approximately $6 \mathrm{~dB}$, see $\mathrm{Ho}$ and Narins 2006 and "Introduction") by the Eustachian tubes or the relatively narrow interaural canal. Additionally, in the frog $R$. pipiens, the eardrums are not as delicate as in many lizards, and the sound transmission through the eardrum and hence the indirect sound component may therefore be attenuated. This is supported by the poorer performance (lower sensitivity, interaural gain, and directionality) of the more superficial eardrum system of Basiliscus.

\section{RECONSTRUCTION AND MODEL}

The reconstructed free-field responses show roughly the same directivity as the free-field measurements, i.e., a relatively large and robust directionality (up to 20-dB difference between ipsi- and contralateral stimulation and generally larger responses from ipsithan contralateral angles). Especially for Hemidactylus, there is a good match between measured and reconstructed directivity. Some of the deviation between measured and reconstructed response are probably due to measurement error. The measurements were carried out on unrestrained, sedated animals, and it is possible that they may have moved slightly during the measurements. Clearly, small movements could obscure the small phase differences between the ears (distance $7-10 \mathrm{~mm}$ in the smaller lizards). The reconstructions seem to be relatively poorer at higher frequencies, where small movements of the animals or differences in positioning the probe microphone will have the largest effects on the phase measurements and the eardrum displacements are much smaller (Manley 1972, 1990), but it is also likely that amplitude and phase differences due to sound diffraction by the lizard will influence the directivity at high frequencies (above $4 \mathrm{kHz}$ ). However, it is also possible that additional inputs, such as lungs or nares, can influence the directivity, even though they are probably much smaller than the tympanic inputs. The lungs vibrate as resonators in the sound field (Hetherington 2001), but for the species investigated here, the resonance frequency is generally below the directional band. Furthermore, unlike the case in frogs (see, e.g., Narins et al. 1988; Jørgensen et al. 1991), the eardrum transfer function does not exhibit a peak at the lung resonance frequency (Christensen-Dalsgaard and Manley 2005), indicating that the lung vibrations are not strongly coupled to the mouth cavity and the tympanum. We have not been able to show any significant effect of blocking the nares in the present experiment (Fig. 2), but it is still possible that sound input from the nares, even if small, could be important for directions close to the midline, where the two tympanal inputs nearly cancel, to steepen the gradient at frontal angles. The very simple lumped-element model of the ear produces the same directivity pattern as observed in the experiments (Fig. 6). Note that the model parameters are not fitted to the data but were actually measured. Therefore, the relatively good fit of the model suggests that the acoustical interaction of the two tympana accounts for most of the directivity of the ear. The impedances calculated from these measurements are comparable to the impedances reported earlier for the tympanum of the frog Rana temporaria (Aertsen et al. 1986), but compared to earlier modeling studies of the frog ear, it is notable how much closer the lizard ear model is to the actual measured response. Since the response of the frog eardrum in a closed coupler could be modeled quite accurately (Palmer and Pinder 1984; Aertsen et al. 1986), the poorer fit of the frog models to the free-field data is likely caused by the acoustic complexity of the frog ear. In frogs, sound probably enters the mouth cavity through the entire head region and through the lungs (Narins et al. 1988; Jørgensen et al. 1991), i.e., sound input is distributed over a large part of the frog's body. Furthermore, as stated above, the interaural coupling is weaker in the frog species investigated than in the lizard species reported here, with an approximately $-6 \mathrm{~dB}$ maximum (Ho and Narins 2006), perhaps because the tympana themselves are less delicate than in most lizards. Therefore, the tympanic inputs may not dominate the response, as they evidently do in lizards at frequencies above $1 \mathrm{kHz}$. It should be noted, however, that both modeling and interaural transmission in frogs have been studied in few ranid species.

Further neural processing of directional information in a lizard has been investigated very recently (Christensen-Dalsgaard, Tang and Carr, unpublished) and shows binaural interaction mainly by contralateral inhibition at the level of the superior olive and nucleus 
magnocellularis in Gekko gecko. The present data strongly suggest that a simple, binaural neural comparison would be very efficient in enhancing binaural differences and directionality in a naturalistic, i.e., free-field setup. The strong directionality shown by gecko midbrain torus semicircularis neurons with free-field stimulation (Manley 1981) could largely be explained by such a simple comparison.

Presumably, the ancestral tympanic ears of most tetrapods formed from spiracles opening directly into the mouth or buccal cavities and were therefore acoustically coupled through the mouth cavity. Therefore, these earliest tympanic ears would have been able to exploit the pressure-gradient directionality (Christensen-Dalsgaard and Manley 2005; ChristensenDalsgaard and Carr 2008). The extreme directionality of the lizard ear shows that the directionality resulting from such an ear can be large and useful for binaural comparisons, and the presumed secondary loss of acoustical coupling in the mammalian ear by formation of closed middle ear cavities may have necessitated additional neural processing, maybe leading to the segregation of time and intensity pathways (ChristensenDalsgaard and Carr 2008). The behavioral implications of the directionality of the lizard ear are unknown, although some lizards are reported to use sound localization for prey detection (Sakaluk and Bellwood 1984) or location of conspecific burrows (Hibbits 2006; Hibbits et al. 2007).

\section{ACKNOWLEDGEMENTS}

This work is supported by grants to JCD from the Danish National Research Foundation and the Danish National Science Foundation and a grant to GAM from the Deutsche Forschungsgemeinschaft.

\section{REFERENCES}

Aertsen AMHJ, Vlaming MSMG, Eggermont JJ, Johannesma PIM. Directional hearing in the grassfrog (Rana temporaria L) II Acoustics and modelling of the auditory periphery. Hear. Res. 21:17-40, 1986.

Christensen-Dalsgaard J. Directional hearing in non-mammalian vertebrates. In: Popper AN, Fay RR (eds) Sound Source Localization. New York, Springer, pp. 67-123, 2005.
Christensen-Dalsgaard J, Carr CE. Evolution of a sensory novelty: tympanic ears and the associated neural processing. Brain Res. Bull. 75:365-370, 2008.

Christensen-Dalsgaard J, Manley GA. Directionality of the lizard ear. J. Exp. Biol. 208:1209-1217, 2005.

Feng AS, Christensen-Dalsgaard J. Interconnections between the ears in non-mammalian vertebrates. In: Basbaum A, Bushnell M, Smith D, Beauchamp G, Firestein S, Dallos P, Oertel D, Masland R, Albright T, Kaas J, Gardner E (eds) The Senses: A Comprehensive Reference, vol 3. New York, Elsevier, pp. 217-224, 2007.

Fletcher NH. Acoustic Systems in Biology. Oxford, Oxford University Press, 1992.

Goldberg JM, Brown PB. Response of binaural neurons of dog superior olivary complex: an anatomical and electrophysiological study. J. Neurophysiol. 32:613-636, 1969.

Hetherington TE. Laser vibrometric studies of sound-induced motion of the body walls and lungs of salamanders and lizards: implications for lung-based hearing. J. Comp. Physiol. A 187:499-507, 2001.

HiввітTs TJ. Ecology and sexual selection of the common barking gecko (Ptenopus garrulus). PhD Thesis, University of the Witwatersrand, Johannesburg, South Africa, 2006.

Hibbitts TJ, Whiting MJ, Stuart-Fox DM. Shouting the odds: vocalization signals status in a lizard. Behav. Ecol. Sociobiol. 61:1169-1176, 2007.

Ho CCK, NARINS PM. Directionality of the pressure-gradient receiver ears in the Northern leopard frog Rana pipiens pipiens. J. Comp. Physiol. A 192:417-429, 2006.

Jørgensen MB, Schmitz B, Christensen-Dalsgaard J. Biophysics of directional hearing in the frog Eleutherodactylus coqui. J. Comp. Physiol. A 168:223-232, 1991.

KLump GM. Sound localization in birds. In: Dooling RJ, Fay RR, Popper AN (eds) Comparative Hearing: Birds and Reptiles. New York, Springer, pp. 249-307, 2000.

Larsen ON, Dooling RJ, Michelsen A. The role of pressure difference reception in the directional hearing of budgerigars (Melopsittacus undulatus). J. Comp. Physiol. A 192:1062-1072, 2006.

MANLEy GA. Frequency response of the middle ear of geckos. J. Comp. Physiol. 81:251-258, 1972.

MANLey GA. A review of the auditory physiology of the reptiles. In: Autrum H-J, Ottoson D, Perl E, Schmidt RF (eds) Progress in Sensory Physiology. Berlin, Springer, pp. 49-134, 1981.

MANLEy GA. Peripheral Hearing Mechanisms in Reptiles and Birds. Heidelberg, Springer, 1990.

Manley GA, Gallo L. Otoacoustic emissions, hair cells and myosin motors. J. Acoust. Soc. Amer. 102:1049-1055, 1997.

Michelsen A, Rohrseitz K. Directional sound processing and interaural sound transmission in a small and a large grasshopper. J. Exp. Biol. 198:1817-1827, 1995.

Moisefr A, Konishi M. The owl's interaural pathway is not involved in sound localization. J. Comp. Physiol. A 144:299-304, 1981.

NARins PM, Ehret G, TAutZ J. Accessory pathway for sound transfer in a neotropical frog. Proc. Natl. Acad. Sci. 85:1508-1512, 1988.

PAlmer AR, PINDER AC. The directionality of the frog ear described by a mechanical model. J. Theor. Biol. 110:205-215, 1984.

SAKALUK SK, BeLLwood JJ. Gecko phonotaxis to cricket calling song: a case of satellite predation. Anim. Behav. 32:659-662, 1984. 